\title{
CUADROS DE JUAN DEL CASTILLO Y ANTONIO DEL CASTILLO EN EL ERMITAGE
}

Juan del Castillo y Antonio del Castillo son dos pintores que trabajaron en Andalucía. Hace algún tiempo se consideraba que Antonio era el sobrino de Juan, actualmente es sabido que no es así, aunque no se excluye que fuesen parientes. $\mathrm{Al}$ ingresar los cuadros de estos pintores en el Ermitage tuvo lugar una confusión debida al parecido de sus apellidos y las obras de uno de ellos se atribuían en los inventarios al otro. Así ocurrió con el pequeño cuadro Visitación ${ }^{2}$ (Fig. 1). Fue adquirido en 1834 a Juan Miguel Páez de la Cadena, embajador de España en San Petersburgo, e incluído en el catálogo manuscrito del museo sin haber indicado el nombre. En el Livret ${ }^{3} \mathrm{y}$ en todos los posteriores catálogos del Ermitage de antes de la revolución, el cuadro fue asignado bajo el nombre de Antonio del Castillo. Según los datos de E.K. Liphart ${ }^{4}$ en el catálogo de 1912 V. von Loga lo atribuyó a Alonso Cano. N. Wrangel tenía dudas de que Antonio del Castillo fuese creador de la tabla, pero no nombró al posible autor ${ }^{5}$. En los catálogos de 1958 y 1976 el cuadro no fue incluido; en el catálogo de 1997 figuró como obra de un pintor anónimo español del siglo XVII, posiblemente, del círculo de Alonso Cano (la composición de esta pintura es similar al cuadro de Cano sobre el mismo tema en el Museo de Goya en Castres, sólo que de mayor tamaño) ${ }^{6}$. A. E. Pérez Sánchez (en opinión expresada a base de fotografía) supuso que su autor pudiese ser Juan del Castillo. Los documentos de archivo recién descubiertos confirma-

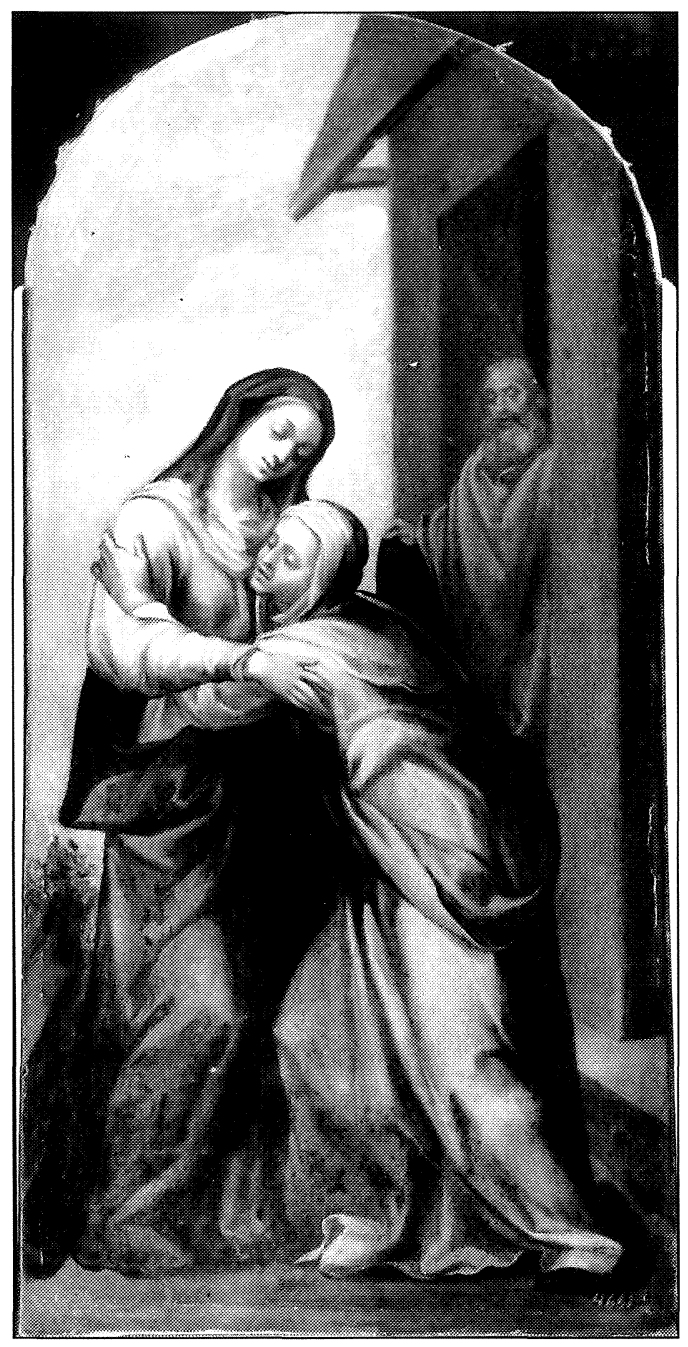

Fig. 1. Juan del Castillo: Visitación.San Petersburgo. Museo del Ermitage.

\footnotetext{
${ }^{1}$ El texto ha sido traducido al castellano por Olga Lipatova. Sobre los cuadros de Antonio del Castillo en el Ermitage fue impartida una ponencia en la conferencia. Cfr. Kagané L.L. Kartini Antonio del Castillo v Ermitazhe. // Ermitazhnie Chteniya pamyati V.F. Levinsona-Lessinga (2.03.1893-27.06.1972). Kratkoye soderzhanie dokladov. Sankt-Peterburg, 2000, s. 17-19 - Kagané L.L. Cuadros de Antonio del Castillo en el Ermitage. // Lecturas del Ermitage dedicadas a la memoria de Levinson-Lessing V.F. (2.03.1893-27.06.1972). Contenido breve de ponencias. San Petersburgo, 2000, pp. 17-19.

${ }^{2}$ Lienzo (transferido de tabla). 55,2 x $30 \mathrm{~cm}$. Inv. 326.

${ }^{3}$ [Labensky F.J.] Livret de la Galerie Impèrial de l'Ermitage de Saint-Pétersbourg, contenant l'explication des tableaux qui la composent, avec de courtes notices sur les autres objets d'art ou de curiosité qui y sont exposés. Saint-Pétersbourg, 1838. En lo sucesivo: Livret 1838.

${ }^{4}$ [Liphart E.K.]. Imperatorskiy Ermitazh. Katalog Kartinnoy galerei. Chast' I. Italianskaya i ispanskaya zhivopis'. [Liphart E.K. Ermitage Impérial. Catálogo de la galería de la pintura. Parte I. La pintura italiana y española. San Petersburgo, 1912. En lo sucesivo Cat. 1912.

${ }^{5}$ Wrangel N. Ob ispanskij kartinaj Ermitazha. // Starie Godi, 1914, yanvar', s. 31. - Wrangel N. Sobre los cuadros españoles del Ermitage. - Años Antiguos, 1914, enero, p. 31.

${ }^{6}$ Kagané L.L. The Hermitage Catalogue of Western European Painting. Spanish Painting. Fifteenth to Nineteenth Centuries. Moscow-Florence, 1997, p. 245, Num. 131. En lo sucesivo Kagané 1997
} 
ron esta suposición. En la lista detallada de cuadros adjunta por Páez de la Cadena durante la venta de la colección, se dice con referencia a esta obra: «Este pequeño cuadro, pintado sobre tabla por la forma del cual se ve que estaba predestinado para una capilla, representa el encuentro de la Virgen con Santa Isabel. Es un cuadro que por su hermosa composición, el dibujo correcto, el colorido, la expresividad de sentimientos, la nobleza de las figuras y asimismo por la exquisita manera de representar los pliegues podría ser la obra de uno de los mejores maestros de la escuela florentina y el cual, sin embargo, pertenece a la escuela de Sevilla y sin lugar a dudas fue ejecutado por Juan del Castillo, maestro de Murillo, uno de los viejos pintores de esta escuela sevillana... Este pequeño cuadro encantador proviene de la colección de los condes de Águila en Sevilla» ${ }^{7}$.

Juan del Castillo (h. 1590-1657) pasó toda su vida en Sevilla. Su obra se formó bajo la influencia de la corriente manierista de la pintura de Sevilla a principios del siglo xvII, más tarde sufrió la influencia de las tendencias realistas tan perceptibles en las obras de sus jóvenes contemporáneos - Velázquez, Zurbarán, Cano-. Juan del Castillo era pariente de Alonso Cano y colaboró mucho con él en la década de 1630. En aquel mismo período daba clases a Bartolomé Esteban Murillo. Los investigadores notan la predisposición de Juan del Castillo a los rostros agradables, a la suavidad y ternura de interpretación de imagenes, a la paleta clara polícroma. Se considera que muchos rasgos de su obra fueron desarrollados más tarde por Murillo.

La comparación del cuadro del Ermitage con otras obras de Juan del Castillo revela una indudable afinidad. En los años 1634-1636 el pintor creó un retablo para la iglesia del Monasterio Montesión en Sevilla (actualmente el retablo se encuentra en el Museo de Bellas Artes en Sevilla). En el cuadro del Ermitage los tipos de rostros, la manera de pintar los pliegues del vestuario son similares a obras como Anunciación y Visitación ${ }^{8}$, aunque los cuadros del retablo son mucho más grandes por su tamaño y están resueltos de un modo más monumental. En la pequeña obra del Ermitage la complicada sinuosidad de la figura de Isabel, el juego caprichoso de los pliegues de su capa evidencian los rasgos indelebles del manierismo. Se puede suponer que este cuadro haya sido creado antes del retablo de Montesión y fecharlo aproximadamente en los años 1630-1635. Como se mencionaba antes según los datos de Juan Miguel Páez de la Cadena el cuadro pertenecía a la colección de los condes de Águila en Sevilla.

Antonio del Castillo (1616-1668) fue el pintor principal de Córdoba a mediados del siglo XVII. La información detallada sobre él fue comunicada por Antonio Palomino, su compatriota y contemporáneo más jóven, autor de biografías de los pintores españoles ${ }^{9}$. Más tarde, durante mucho tiempo, no aparecieron investigaciones sobre la obra de Antonio del Castillo, éste comenzó a atraer la atención hacia su persona desde la década de 1960. P.Muller prestó una atención especial a los dibujos del pintor. También J.Valverde Madrid estudió y dio un analisis profundo de la obra de Antonio del Castillo. Últimamente M. Nancarrow Taggard se ha ocupado de la obra del pintor. Asimismo hay que acordarse del libro de B. Navarrete sobre la in-

\footnotetext{
${ }^{7}$ Rossiyskiy Gosudarstvenniy Istoricheskiy Arkhiv (RGIA), El Archivo Histórico Estatal Ruso (AHER), fondo 469, inventario 8,1834, expediente 177. Notice raisonnée ou observations et éclairssement sur la note, ci jointe, de la petite collection de tableaux. (En lo sucesivo - Notice 1834). L. 10, num. 28: «Ce petit tableau peint sur bois, dont la forme annoncer avoir été destiné á une chapelle et que represente la visitation de la sainte Vierge á Ste. Elisabeth. Tableau qui d’aprés sa belle composition, son dessin si correct, son coloris, l'expression si bien sentu et si noble des figures, et la belle manière dont elles sont draperis, pouvait bien passer pour être d'un des premières maîtres de l'école de Florence et qui pourtant n'est que de l'école de Seville, est indubitablement de la main de J.Castillo, maître de Murillo et l'un des anciens maîtres de cette école de Seville... Ce charmant petit tableau, vient de la colection de comtes del Aguila à Seville».

${ }^{8}$ Cfr. E. Valdivieso, J.M. Serrera, Historia de la pintura sevillana del primer tercio del siglo XVII. Madrid, 1985, láms. $219,221$.

${ }^{9}$ Palomino A., El museo pictórico y escala óptica. III. El Parnaso español pintoresco laureado. Madrid, $1715-1724$ (Ed. 1947, p. 296). En lo sucesivo Palomino 1724.
} 
fluencia del grabado en la pintura sevillana donde se mencionan las analogías relacionadas con las obras del maestro ${ }^{10}$.

Antonio del Castillo nació en Córdoba en la familia de un pintor, se quedó huérfano muy temprano, aprendió la pintura en su ciudad natal y después en Sevilla (el tiempo de su estancia allí se desconoce). La obra de Zurbarán ejerció una gran influencia en Antonio del Castillo, quien estudió también el grabado flamenco. Desde el año 1635 el pintor se instaló para siempre en Córdoba. Antonio del Castillo creaba composiciones grandes y monumentales para la catedral y las iglesias en una manera bien acusada de claroscuro, dura y seca. Paralelamente hizo muchos dibujos del natural representando escenas de la vida campestre. Según Palomino, Antonio del Castillo prefería paisajes y retratos que se apreciaban mucho por los amantes del arte. Los resultados de la observación del mundo circundante se reflejaron no sólo en los dibujos del maestro sino en la serie de cuadros creados por él y dedicados al patriarca José (Museo del Prado, Madrid). Nancarrow Taggard ${ }^{11}$ observó los cambios de estilo del pintor a mediados de la década de 1650. En su pintura aparecieron los fondos de paisaje, se suavizaron los contornos, la pincelada se hizo más amplia y libre. Esto se reveló más claramente en la serie de cuadros de la vida de José en los que el pintor no seguía tan fielmente la tradición como en las obras religiosas destinadas para las iglesias.

En el Ermitage se guarda una pequeña obra de Antonio del Castillo Cargando los fardos ${ }^{12}$ (Fig. 2). Ingresó con la atribución a este pintor en 1814 formando parte de la colección de W. Coeswelt, se mencionaba con su mismo nombre en el Livret ${ }^{13}$ pero después fue entregada al Palacio Tavricheskiy y de allí en 1860, al Palacio de Gatchina y no fue incluida en los catálogos del Ermitage de antes de la revolución. En el año 1926 el lienzo fue devuelto al Ermitage. Por primera vez la obra fue descrita en el artículo de Muller. Ésta comparó el cuadro del Ermitage con el dibujo de Antonio del Castillo de igual tema (Academia San Fernando, Madrid), que tiene la firma del maestro y así confirmó la pertenencia de ambas obras al mismo pintor ${ }^{14}$ (Fig. 3). Muller leyó la etiqueta pegada en el reverso del lienzo donde erróneamente se mencionaba el nombre de Juan del Castillo y decidió que era ella quien por primera vez atribuyó la obra a Antonio del Castillo, mientras que dicha pintura siempre llevó el nombre de este pintor. Valverde calificó el cuadro del Ermitage como «una obra magnífica» e indicó otros dos lienzos sobre el asunto campesino, ambos bajo el nombre Cabaña que se encontraban en la colección sevillana de López Cepero y en la colección de Cortés Pujadas en Córdoba ${ }^{15}$. Pérez Sánchez, que mencionó el cuadro del Ermitage con relación a la investigación de los dibujos del maestro, tampoco dudaba de su autenticidad ${ }^{16}$. La única persona que puso en duda su autenticidad fue Nancarrow Taggard ${ }^{17}$. En su opinión algún otro pintor hizo la copia pictórica del dibujo del

\footnotetext{
${ }^{10}$ Muller P., «Antonio del Castillo and the Rustic Style». Apollo, 1966. November, p. 380 y s. En lo sucesivo Muller 1966; Valverde Madrid, J., «El pintor Antonio del Castillo», Boletín de la Real Academia de Córdoba, 1961, año 32, núm. 82, pp. 5-279; Valverde Madrid, J., «Nuevos datos sobre el pintor Antonio del Castillo», Boletín de Bellas Artes de la Academia sevillana, 1976, pp. 197-260. En lo sucesivo Valverde Madrid 1976; Nancarrow Taggard, M., «Narrative meaning in Antonio del Castillo's "The Life of Joseph"», Gazette des Beaux-Arts (GBA), t. 116, 1990, october pp. 116-128; Nancarrow Taggard, M., «New Dates and Documentation for Antonio del Castillo's Paintings in Cordoba Cathedral», GBA, t. 120, 1992, novembre, pp. 165-173; Nancarrow Taggard, M., «Antonio del Castillo: correspondencia entre dibujos de escenas rurales e "historiejas"», Goya, núms. 235-236, 1993, pp. 65-76. En lo sucesivo Nancarrow Taggard 1993; Navarrete Prieto, B., La pintura andaluza del siglo xvI y sus fuentes grabadas. Madrid, 1998, pp. 172, 175. En lo sucesivo Navarrete 1998.

"Nancarrow Taggard, 1993, pp. 65-76.

${ }^{12}$ Lienzo, $36 \times 28 \mathrm{~cm}$. Inv. 2572 .

${ }^{13}$ Livret, 1838 , p. 427.

${ }^{14}$ Muller 1966, p. 380 y s.

${ }^{15}$ Valverde Madrid, 1976, pp. 202, 218-219, núm. 104.

${ }^{16}$ Pérez Sánchez, A.E. El dibujo español de los siglos de oro. Salas de Exposiciones del Palacio de Bibliotecas y Museos, Madrid, Mayo 1980, p. 59.

${ }^{17}$ Nancarrow Taggard, 1993, p. 70.
}

AEA, LXXVI, 2003, 302, pp. 187 a 205 

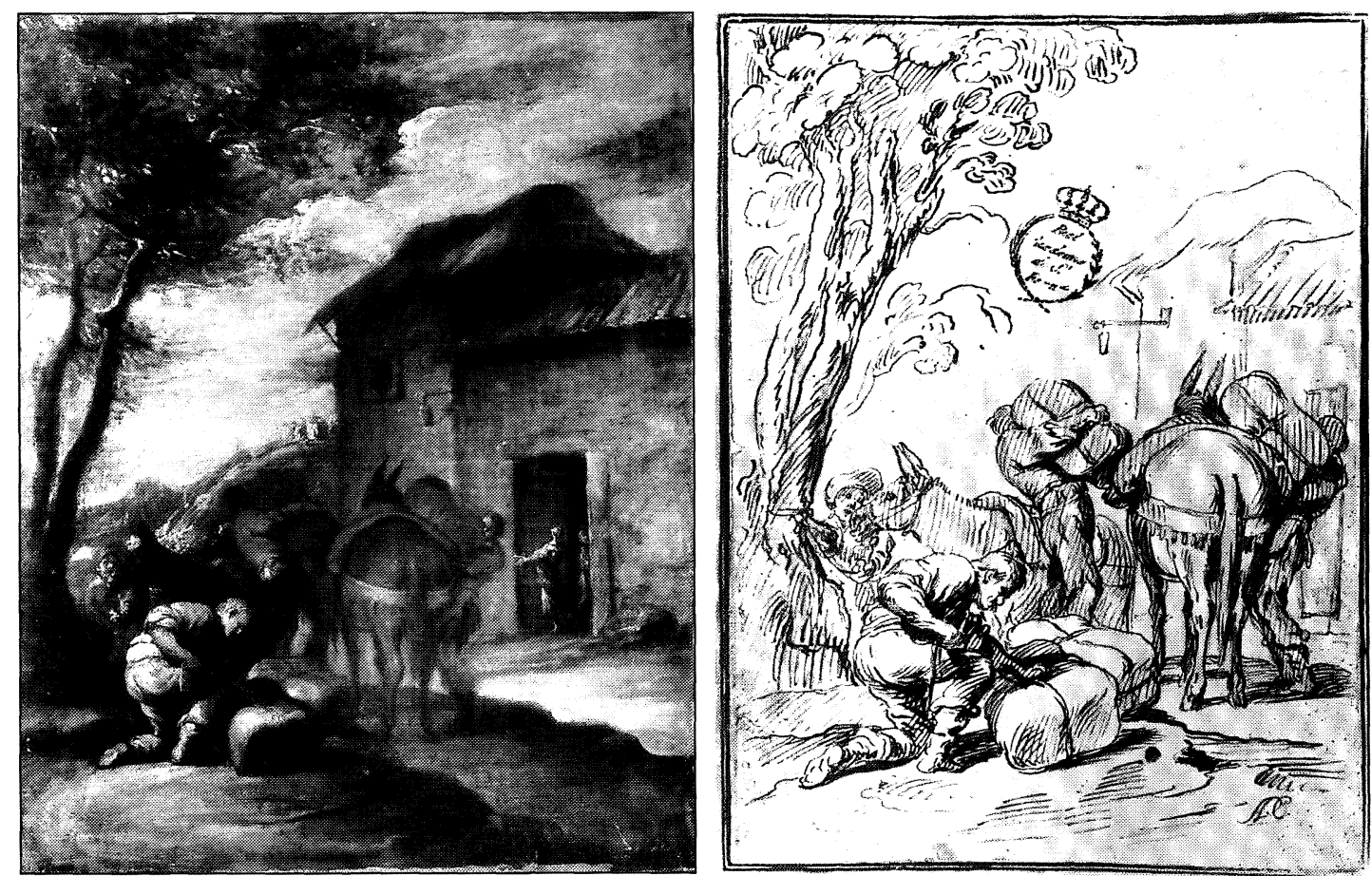

Figs. 2 y 3. Antonio del Castillo: Cargando los fardos. Óleo (San Petersburgo. Museo del Ermitage) y dibujo (Madrid. Academia de San Fernando).

maestro. Los argumentos suyos se reducen, primero, a que en aquel entonces en España no había clientes que tuvieran interés por los cuadros sobre los temas campesinos y, segundo, el estilo pictórico de la obra no correspondía a las pinturas creadas por este maestro. Sin embargo, hay que prestar atención a las palabras de Palomino: «Fue también nuestro Castillo excelente paisista, para lo cual se salia algunos días a pasear con recado de dibujar, y copiaba algunos sitios por el natural» ${ }^{18}$. Dichas palabras permiten llegar a la conclusión de que Castillo hacía los dibujos para después a base de ellos crear sus obras pictóricas. Además, Palomino añadia que «en muchas casas de Córdoba hay sus paisajes». Navarrete demostró convincentemente que algunos detalles de los dibujos de Castillo están inspirados en los grabados de Boetius a Bolswert hechos según los cuadros de Bloemaert ${ }^{19}$. Es también una prueba de que los asuntos sobre la vida campesina ya estaban de moda en Sevilla en la segunda mitad del siglo XVII.

Precisamente Nancarrow Taggard llamó la atención de cómo la manera pictórica iba cambiando notablemente en los cuadros del pintor de la seca y lineal a la más libre con una pincelada fluída y una variedad de matices de color. El dibujo similar al cuadro del Ermitage se fecha en la mitad de la década de 1660. En esos momentos se fecha también el cuadro. Es de notar que la pintura de este cuadro comparada con otras labores del maestro se destaca por un virtuosisimo extraordinario. Las figuras menudas que están cerca de la puerta revelan la mano de un dibujante magnifico, tal como era Castillo, pero más que todo sorprende el brío de las pinceladas. La interpretación del follaje de los árboles no dibujados detalladamente sino puestos en masa con golpes separados del pincel es semejante a la impresionista. Es probable que este pequeño cuadro sea uno de los más tardíos en la obra del maestro y refleja sus logros supremos en la pintura.

${ }^{18}$ Palomino, 1724 (Ed. 1947, p. 296).

${ }^{19}$ Navarrete, 1998, pp. 172, 175. 

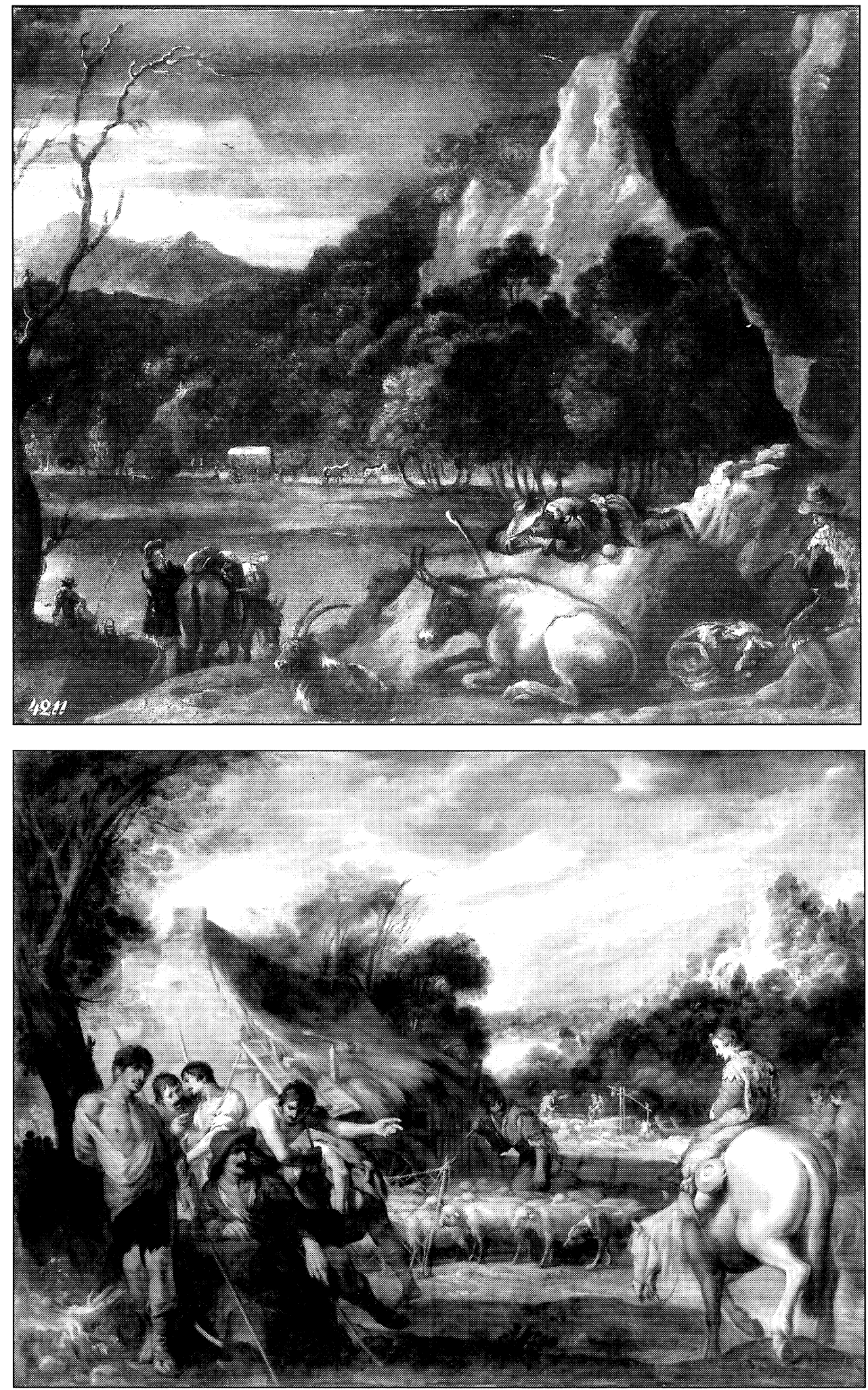

Fig. 4. Antonio del Castillo: Paisaje montuoso. San Petersburgo. Museo del Ermitage. Fig. 5. Antonio del Castillo: José y sus hermanos. Madrid. Museo del Prado.

AEA, LXXVI, 2003, 302, pp. 187 a 205 
Hay razones para vincular el Paisaje montuoso ${ }^{20}$ (Fig. 4) con la obra de Antonio del Castillo. Asimismo ingresó junto con la colección de W. Coeswelt en 1815 pero figuraba como obra de Francisco Herrera el Mozo. Probablemente, abrigando dudas de lo correcto de esta atribución, G. Waagen no incluyó esta pintura en su libro dedicado al Ermitage. Posteriormente no entró en los catálogos del Ermitage, ni en la literatura, mas en el inventario está presente como la obra de un pintor español anónimo del siglo XVII. En el catálogo de 1997 fue devuelto el nombre de Herrera el Mozo pero con interrogación ${ }^{21}$.

En el cuadro está representado un arroyo en las montañas y los pastores con el ganado. Abundan los detalles de la vida cotidiana. En el centro se ven los animales, una cabra y una vaca, a la izquierda está un arriero junto al mulo cargado de fardos, seguido por un pescador con caña de pescar, tras el arroyo por el camino avanza un carro enganchado por cuatro caballos. Es necesario recordar las palabras de Palomino de que Antonio del Castillo dibujaba del natural los alrededores, incluyendo «cabañas y cortijos de aquella tierra, donde copiaba también los animales, carros y otros adherentes que hallaba a mano; y algunas casualidades en aquel arroyo de las peñas con singularísimo primor...» ${ }^{22}$. Estas palabras, sobre todo la mención «del arroyo de las peñas», parecen referirse directamente al cuadro del Ermitage. Compárandolo con otras obras del maestro atraen la atención los rasgos de semejanza en los detalles. El pastor yacente sobre la piedra podría estar tomado de la Escena campestre de Cornelius de Bloemaert grabada por Boetius a Bolswert. Navarrete vió el parecido de este mismo personaje con el dibujo de Antonio del Castillo Escena campestre del Museo del Prado ${ }^{23}$. Pero en el cuadro del Ermitage el parecido es más acusado. El hombre del sombrero de alas anchas, vestido de caftán y de botas de montar, tocando la flauta, que está sentado a la derecha en el paisaje del Ermitage, se parece a los participantes de las escenas en los dibujos del maestro. Sin embargo lo que es más característico de Antonio del Castillo es la representación de los animales. El mulo cargado que está vuelto de grupa al espectador es un motivo que se encuentra con mucha frecuencia en los dibujos del pintor y está presente también en el cuadro del Ermitage Cargando los fardos. La vaca yacente igual que la del Paisaje montuoso está representada en el dibujo Escena campestre (Academia San Fernando, Madrid). El fondo del paisaje también tiene analogías en la obra del pintor. En uno de los cuadros sobre los asuntos bíblicos, dedicado a José, precisamente en José y sus hermanos (Museo del Prado, Madrid) (Fig. 5) están representadas análogamente las rocas blancas que se alzan por encima de la copa de los árboles. El plano alejado con figuras menudas recuerda el camino por donde pasa el furgón en el cuadro del Ermitage. Al mencionar los rasgos de semejanza del Paisaje montuoso con los dibujos y las obras pictóricas del maestro, hay que notar que está pintado más tímidamente que los cuadros Cargando los fardos y José y sus hermanos. Aquí se puede hablar sólo de las tendencias hacia una pincelada libre en detalles tales como las figuras de los pastores en el primer término a la izquierda; la cara del mulero, que recuerda el rostro de uno de los personajes del segundo plano en el cuadro Cargando los fardos; el carro de caballos, las pendientes de la montaña, el follaje. Si admitimos que el autor del Paisaje montuoso es Antonio del Castillo, en tal caso será una de las primeras pruebas del maestro, que es conocido por su obra religiosa, de recurrir a la pintura de género. El paso del pintor al nuevo estilo se produce a mediados de 1650. El Paisaje montuoso podría ser creado hacia esa fecha.

El Paisaje con cabañas ${ }^{24}$ (Fig. 6) fue adquirido de Juan Miguel Páez de la Cadena en

\footnotetext{
${ }^{20}$ Lienzo. $50 \times 62 \mathrm{~cm}$. Inv. 1453.

${ }^{21}$ Kagané, 1997, p. 96, núm. 33. La ilustración está puesta errónea en la p. 249.

${ }^{22}$ Palomino, 1724, ed. 1947 , p. 296.

${ }^{23}$ Navarrete, 1998, p. 175.

${ }^{24}$ Lienzo. $60,5 \times 80,5 \mathrm{~cm}$. Inv. 317 .
} 

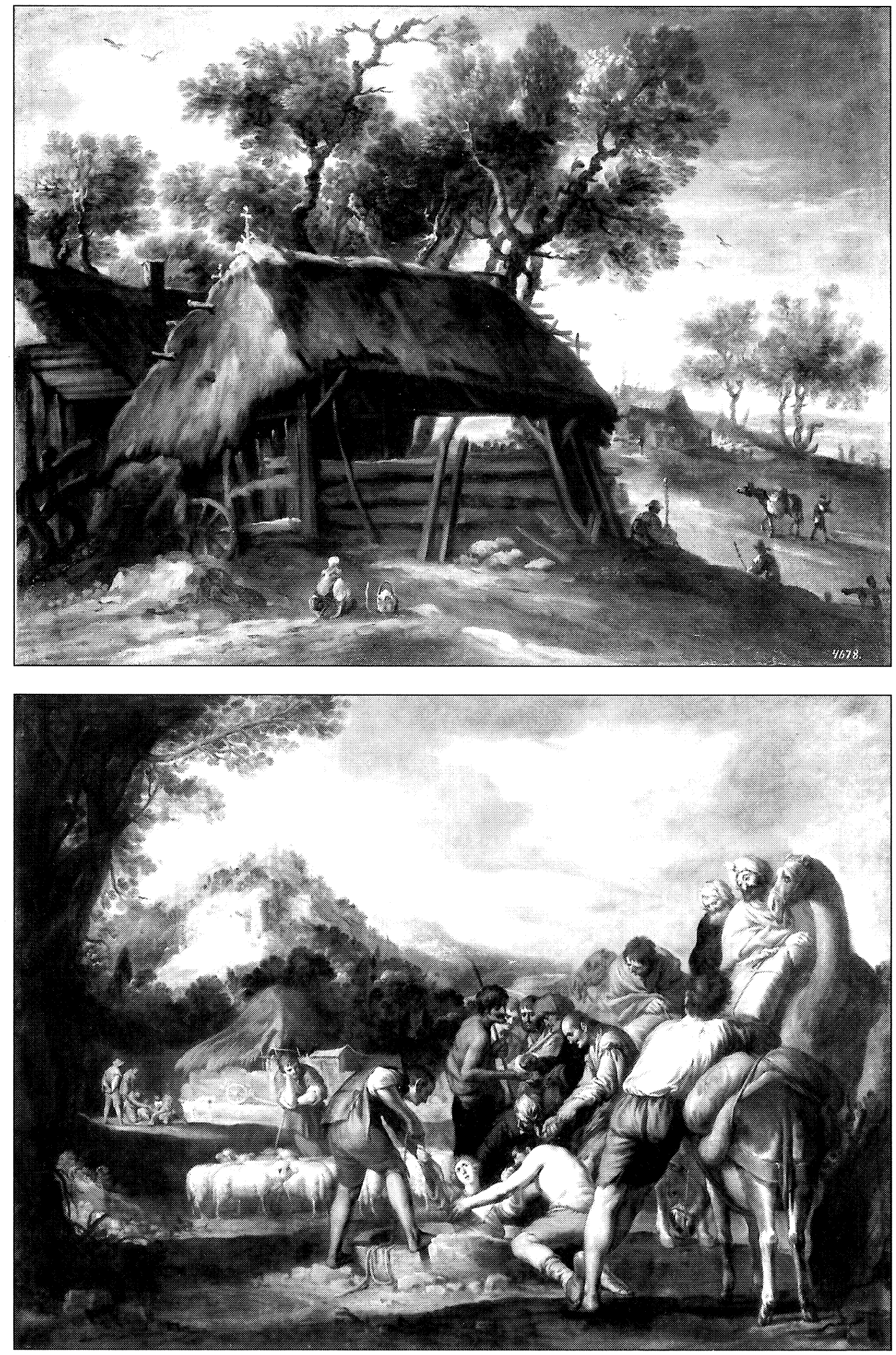

Fig. 6. Antonio del Castillo: Paisaje con cabañas. San Petersburgo. Museo del Ermitage. Fig. 7. Antonio del Castillo: José vendido por sus hermanos. Madrid. Museo del Prado.

AEA, LXXVI, 2003, 302, pp. 187 a 205 
1834 como una obra de Velázquez ${ }^{25}$. Según los datos del propietario el cuadro se hallaba antes en la colección de Gaspar Melchor de Jovellanos, famoso estadista y mecenas del arte. Con el nombre de Velázquez el cuadro fue inscrito en el Livret. Sin embargo, L.Viardot se negó a reconocer su autoría y Waagen la puso en duda ${ }^{26}$. En el catálogo de 1863 el paisaje figura como «atribuido a Velázquez», en el de 1891 está presentado como de Velázquez (?), en el catálogo de 1912 Liphart lo inscribió entre las obras de los pintores anónimos españoles del siglo xvII, comunicando que, en la opinión de E.Tormo y V. von Loga, el autor del cuadro es un pintor de la escuela sevillana ${ }^{27}$. El paisaje representa un tema de la vida campestre, típico de Antonio del Castillo y hay bastantes razones para relacionar esta obra con su nombre. Las cabañas con tejadillo de paja, detalle asimilado por Castillo de los grabados de Boetius a Bolswert, se encuentra constantemente en los dibujos del pintor y también en los cuadros sobre los temas de la historia del patriarca José. En el dibujo La mujer con un niño en brazos (Academia San Fernando, Madrid) el rincón de la cabaña donde está la rueda, arrimada a la pared, es idéntico al del cuadro del Ermitage. Un detalle análogo llama la atención en el cuadro de Antonio del Castillo José vendido por sus hermanos (h. 1655, Museo del Prado, Madrid) ${ }^{28}$ (Fig. 7), el parecido se observa también en la tratamiento del follaje de los árboles. Navarrete subrayó que Castillo había asimilado la representación de los tejados de paja y de los árboles de los grabados de Bolswert hechos según los cuadros de Bloemaert ${ }^{29}$. En el cuadro del Ermitage esta asimilación se nota muy claramente. En la representación de los planos alejados hay parecido con el cuadro de Antonio del Castillo perteneciente a la serie del patriarca José, precisamente el cuadro José y sus hermanos. Así, pues, con mayor razón se puede atribuir el Paisaje con cabañas a Antonio del Castillo fechándolo aproximadamente con el tiempo de la creación de los cuadros de la serie de la vida de José, es decir, en la mitad de los años 50 del siglo xviI.

Hace falta señalar que entre el Paisaje con cabañas y el Paisaje montuoso existe una relación directa. En ambos cuadros el pescador que está sentado con caña de pescar a la orilla del arroyo es idéntico. Según la manera de ejecución los cuadros se diferencian. El Paisaje con cabañas está pintado con más soltura y maestría. Por eso parece más al cuadro Cargando los fardos, aunque no alcanza el nivel de virtuosismo de este pequeño lienzo. Si aceptamos la autoría de Antonio del Castillo en lo que toca a los cuadros Paisaje montuoso y Paisaje con cabañas resulta que el Ermitage posee tres cuadros raros del pintor, conocido por su interés hacia la vida campestre dibujándola del natural. Estos cuadros dan una idea sobre la evolución de la obra del maestro. El Paisaje montuoso se fecha a fines de 1640 y principios de 1650. El Paisaje con cabañas se dataría a mediados de 1650 y Cargando los fardos a 1660. Asimismo se puede observar cómo iba perfeccionándose la maestría pictórica del artista logrando los mayores éxitos al final de su obra.

\section{KAGANÉ \\ Museo del Ermitage}

\footnotetext{
${ }^{25}$ Notice 1834: «N. ${ }^{\circ} 45$. Ce tableau peint sur toile qui represent un paysage avec quelques cabanes, quelque figures ebauchées des arbres est aussi indubitablement de la main hardu et de la touche savante de Velazquez. On y voit son savoir fuire, le talent de la perspective et la magie de peindre l'atmosphère. Velazquez n'a peint que trés peu de paysages, ils sont trés rares' il n'en a fait que dans ses différents voyages en Italie et imitant comme dans celui ci la manière et le ton de coloris de l'école de Venise. Ce tableau vient aussi de la belle collection de Monsieur de Jovellanos.»

${ }^{26}$ Livret, 1838, p. 419; Viardot L. Les musées d'Allemangne et de Russie. Paris, p. 63; Waagen G.F. Die Gemäldesammlung in der Kaiserlichen Ermitage zu St. Petersburg nebst Bemerkungen über andere dortige Kunstsammlungen. München, 1864. S. 107.

${ }^{27}$ Koehne, B. de, Ermitage Impérial. Catalogue de la Galerie de tableaux. Saint-Pétersbourg, 1863, N. ${ }^{\circ} 425$; Brüiningk E., Somoff A. Ermitage Impérial. Catalogue de la Galerie des tableaux. Vol. I: Les Ecoles d'Italie et d'Espagne. SaintPétersbourg, 1891, N. ${ }^{\circ} 425$; Cat. 1912, N. ${ }^{\circ} 425$.

${ }^{28}$ Agradezco a F. Checa Cremades y a P. Silva Maroto quienes amablemente me ayudaron a ver las historiejas de Antonio del Castillo en el depósito del museo del Prado.

${ }^{29}$ Navarrete, 1998, p. 172.
} 